\title{
A Campaign for the Detection of Earth-Mass Planets in the Habitable Zone of Alpha Centauri
}

\author{
Robert A. Wittenmyer ${ }^{1}$, Michael Endl ${ }^{2}$, Christoph Bergmann ${ }^{3}$, \\ John Hearnshaw ${ }^{3}$, Stuart I. Barnes ${ }^{2,3}$ and Duncan Wright ${ }^{1}$ \\ ${ }^{1}$ Department of Astrophysics, School of Physics, Faculty of Science, The University of New \\ South Wales, 2052, Australia \\ email: rob@phys.unsw.edu.au \\ ${ }^{2}$ McDonald Observatory, University of Texas at Austin, \\ Austin, TX 78712, USA \\ ${ }^{3}$ Department of Physics and Astronomy, University of Canterbury, Christchurch 8011, \\ New Zealand
}

\begin{abstract}
We review the possible formation and orbital stability of Earth-mass or super Earthmass planets around either of the stars Alpha Centauri A or B and describe a program at Mt John University Observatory using the Doppler method that aims to detect such planets. From New Zealand, we are able to observe the Alpha Centauri system year-round. This is critical in order to acquire data of sufficient quantity and phase coverage to detect the orbit of a terrestrial-mass planet in the habitable zone. Our observations are being made at high resolution $(R=70,000)$ and high signal-to-noise with the Hercules vacuum echelle spectrograph attached to the 1-m McLellan telescope by a 25-m long optical fibre and using an iodine cell. We discuss the velocity precision and instrumental stability required for success and outline the progress of the observations so far. At present we are collecting about 10,000 observations of each star, A and $\mathrm{B}$, per year with a typical precision of $2.5 \mathrm{~m} / \mathrm{s}$ per observation.
\end{abstract}

Keywords. stars: individual ( $\alpha$ Centauri), (stars:) binaries: spectroscopic, techniques: radial velocities, methods: data analysis

\section{Introduction}

We are performing the most intensive search to date for Earth-like planets orbiting the nearest stars, Alpha Centauri A and B. The search for a true Earth-analogue planet is one of the boldest scientific and intellectual endeavours ever undertaken by humankind. The successful discovery of a terrestrial planet orbiting in the habitable zone of a nearby Sun-like star will have an unprecedented cultural as well as scientific impact. A first glimpse at the frequency of terrestrial planets in the habitable zones of other stars will have a direct impact on our understanding of the formation of rocky planets and the abundance of habitable worlds in our Galaxy.

Radial-velocity planet detections have been steadily pushing toward ever-lower masses in the 20 years since the first major planet search efforts began. At first, only Jupiter-mass gas-giant planets were detectable (e.g. Murdoch et al. 1993, Walker et al. 1995), but now, with radial-velocity precision approaching $1 \mathrm{~m} / \mathrm{s}$, "super-Earths" are being discovered with masses below 10 Earth-masses (Vogt et al. 2010, Mayor et al. 2009). Models of planet formation predict a vast number of terrestrial-mass planets (e.g. Ida \& Lin 2004, Zhou et al. 2005), which are just now becoming detectable. The radial-velocity signal of 
an Earth-mass planet in a 1 AU orbit is only $\sim 0.1 \mathrm{~m} / \mathrm{s}$. However, with a large enough number of measurements, even signals below the individual measurement uncertainty can be detected with high significance (Narayan et al. 2005, Cochran \& Hatzes 1996). We are now taking the next step in the radial-velocity search for extrasolar planets, extending the search to Earth analogues.

Owing to the extreme observational effort required (tens of thousands of measurements of a single target) and the shortage of time on large telescopes, the current major planetsearch groups cannot afford such an approach. However, a dedicated radial-velocity program with a precision of $3 \mathrm{~m} / \mathrm{s}$ could detect an Earth-analogue in the Alpha Centauri system in 3-5 years (Guedes et al. 2008). We are now undertaking an intensive observing campaign of the nearest Sun-like stars, Alpha Centauri A and B, to search for terrestrial planets in their habitable zone. We will either discover an Earth-like planet orbiting one of the nearest stars, or we will definitively rule out such planets.

\section{Approach and Methodology}

\subsection{Observations}

All observations for this program are performed using the Mt John University Observatory 1-metre telescope located near Lake Tekapo, New Zealand. The HERCULES spectrograph (Hearnshaw et al. 2002), with a resolving power of 70,000, is enclosed in a vacuum tank, minimising shifts due to temperature and pressure changes. The Mt John/HERCULES system achieves radial-velocity precision better than $3 \mathrm{~m} \mathrm{~s}^{-1}$ for bright stars like Alpha Centauri A \& B. Key to the success of this project is the availability of sufficient telescope time. Since 2009, we have obtained an average of $\sim 120$ nights per year. As of 2012 October, we have obtained 24,622 spectra of Alpha Centauri A, and 17,375 spectra of B. Precision radial velocities are computed using the Austral Doppler reduction pipeline (Endl et al. 2000), a proven Doppler code which has been used in several other planet-search programs (e.g. Kürster et al. 2000, Wittenmyer et al. 2011, Endl et al. 2012, Robertson et al. 2012). The special case of the close binary Alpha Centauri system has also prompted the development of an Austral version capable of fitting double-lined spectra - further details are given elsewhere in this volume (Bergmann et al.).

\subsection{Demonstrated Capabilities: Pilot Study}

From 2007-2009, we performed a first pilot study to observe Alpha Centauri with Hercules. Over 7 observing runs and 28 nights we observed Alpha Centauri A and B. Preliminary analysis of the data with our Austral radial-velocity code shows that we already achieved a long-term radial-velocity precision of $2.8 \mathrm{~m} \mathrm{~s}^{-1}$ for component A (see Figure 1). About $4 \%$ of the data were rejected as outliers by a simple $\sigma$-clipping routine (which has the effect of removing all poor S/N-ratio spectra). In 2009 April we had an intensive 10 night long observing run to simulate the monthly observing runs typical for this project. Over these 10 nights we collected a total of 963 spectra of Alpha Centauri A. The data have an overall rms scatter of $2.68 \mathrm{~m} \mathrm{~s}^{-1}$, after removal of a linear slope due to the binary orbit. These results are very similar to radial-velocity results for Alpha Centauri A from the ESO 3.6m/HARPS (Bazot et al. 2007), VLT/UVES and AAT/UCLES (Butler et al. 2004). The nightly rms values given for the HARPS data in Bazot et al. range from 1.80 to $3.26 \mathrm{~m} \mathrm{~s}^{-1}$ for 5 nights, quite comparable to our Hercules results (1.87 to 3.54 $\mathrm{ms}^{-1}$ for 8 nights). The HERCULES spectrograph is enclosed in a vacuum tank, and has no moving parts; the result is that Hercules achieves superb long-term radial-velocity 

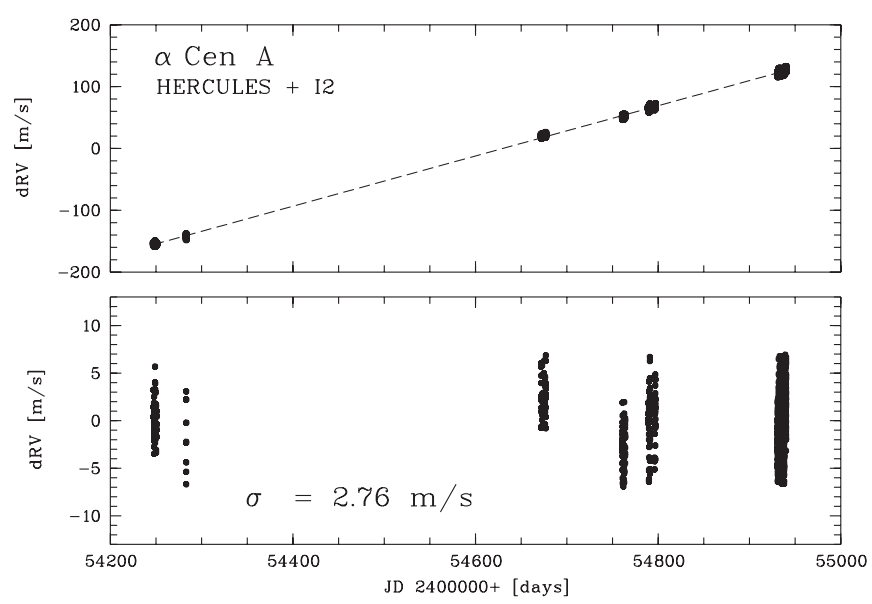

Figure 1. Top: Hercules radial-velocity data for Alpha Centauri A from our pilot study. Bottom: Same, with the binary orbit removed. Over a 2-year period, we achieve a velocity rms scatter of only $2.76 \mathrm{~m} \mathrm{~s}^{-1}$ demonstrating the excellent stability of the Hercules spectrograph.

stability (Hearnshaw et al. 2002, 2009). The results from this preliminary work indicate that the critically necessary long-term velocity precision is feasible.

\subsection{Intrinsic Stellar Variability}

The biggest challenge to perform this kind of search arises from the intrinsic variability of stars themselves. This radial-velocity variability, often dubbed "jitter," encompasses all kinds of velocity and contrast changes in the photosphere of the observed star. The most common type of jitter is a radial-velocity signal produced by rotational modulation due to photospheric spots. For active stars, this type of intrinsic variability can mimic the radial-velocity signature of a short-period planet (e.g. Queloz et al. 2001). Another type of jitter is stellar pulsation, both radial as well as non-radial. For Sun-like stars, pmode oscillations have periods of only a few minutes and they can be effectively removed from the radial-velocity data by binning observations over the pulsation cycle (O'Toole et al. 2009b). This approach was instrumental in the discovery of three low-mass planets orbiting 61 Vir (Vogt et al. 2010). Moreover, the pulsation spectra of Alpha Centauri A \& B have already been characterised by Bedding et al. (2004) and Kjeldsen et al. (2005). So far, these two types of jitter do not pose significant problems to our program as the time scales are 1) well-known, and 2) much shorter than the orbital periods of planets in the habitable zone.

The most serious problem for an Earth-analogue radial-velocity search comes from jitter related to slowly-changing mean granulation patterns over the magnetic cycles of the stars, similar to the Sun's 11-year cycle. Over a magnetic cycle, small changes in the balance between rising and falling granules can lead to a slow change in the mixture of red-shifted and blue-shifted parts of the stellar absorption lines. This can show up as a small radial-velocity drift that could be misinterpreted as an orbiting companion. Ayres (2009) analysed 30 years of X-ray data on the Alpha Centauri system, and showed that both stars have activity cycles that last about 10 years. Although the time scale of the magnetic cycles appears to be much longer than 1 year, we will use the available activity indicators like $\mathrm{H} \alpha$ and the $\mathrm{Ca}$ II $\mathrm{H} \& \mathrm{~K}$ lines (both are well-known proxies for the activity level of stars) to check for any correlations with our radial-velocity data. In summary, the jitter time scales for the Alpha Centauri system are well-understood, significantly 
different from the orbital periods of planets in the habitable zone, and will not lead to a confusion of planet/jitter-related radial-velocity signatures.

\section{Significance and Innovation}

Since Alpha Centauri is the nearest binary star system, the presence or absence of planets will answer a key question about the formation of planets in binary systems. That is: Can terrestrial planets form in close binary star systems? This is significant because nearly $60 \%$ of Sun-like stars in the solar neighbourhood are members of multiple systems (Duquennoy \& Mayor 1991). The Alpha Centauri system is our closest neighbour in space $(\mathrm{d}=4.3$ light years $)$ and thus constitutes a target of fundamental importance for any exoplanet search program. Alpha Centauri A is a star very similar to our Sun, and Alpha Centauri B is slightly cooler and fainter. Their binary orbital period is about 80 years, and the minimum separation of the two stars is about 12 AU (Heintz 1982, Pourbaix et al. 1999).

The formation of terrestrial planets in close binary systems such as Alpha Centauri has been modelled by Quintana et al. (2002, 2007), Quintana \& Lissauer (2006), and Guedes et al. (2008). These studies suggest that the formation of several Earth-mass planets at separations less than $2 \mathrm{AU}$ is possible in the perturbing environment of the stellar companion. Simulations of a binary star system with a giant planet (Haghighipour \& Raymond 2007) showed that water-rich terrestrial planets can form in the habitable zones of such binary systems. Guedes et al. (2008) also simulated the radial-velocity detectability of these planets using synthetic radial-velocity data sets. They demonstrate that a 1.7 Earth-mass planet with a period of 1.2 years can be clearly detected around Alpha Centauri B using 3 years of observations, if the noise distribution is sufficiently close to Gaussian. Thebault et al. (2008, 2009), however, argue that Earth-like planets might not have formed around Alpha Centauri B because the mutual velocities of planetesimals were too high to allow accretion and continued growth to proto-planets beyond the planetesimal phase. Xie et al. (2010) and Xie et al. (2011) expanded on the simulations of Thebault et al. (2009) by starting with km-sized objects (rather than Moon-sized protoplanets), and by allowing them to orbit at inclinations up to $10^{\circ}$ outside of the binary orbital plane. These much more realistic simulations indicated that terrestrial-mass planets can form within $\sim 2$ AU of Alpha Centauri B. There remains some controversy as to whether Earth-like planets could have formed in the Alpha Centauri system. This study is therefore significant because the results will robustly establish either the presence or absence of terrestrial-mass planets in this important nearby star system.

This project is significant because it can be regarded as the first ultra-deep reconnaissance of Sun-like stars in the solar neighbourhood, an important step in preparing future space missions to directly detect these planets. While NASA's Kepler mission has given us valuable data on the frequency of habitable planets in our region of the Galaxy, the Kepler stars are at a distance of several hundred parsecs. This makes further follow-up observations (in particular, the critical radial-velocity observations which provide dynamical masses) extremely difficult even for the largest existing telescopes (Borucki et al. 2010, Gautier et al. 2010). The same is true for the on-going microlensing surveys (e.g. Gould et al. 2006) which are capable of finding true Earth-analogues (although around very distant stars). Such lensing systems tend to be kiloparsecs away, making follow-up observations essentially impossible. Any future planet detection mission which will attempt to obtain a direct image of a planet using an orbiting coronagraphic telescope or an array of infrared telescopes will concentrate on the nearest stars, where projected separations are larger. Of these four major techniques to search for 

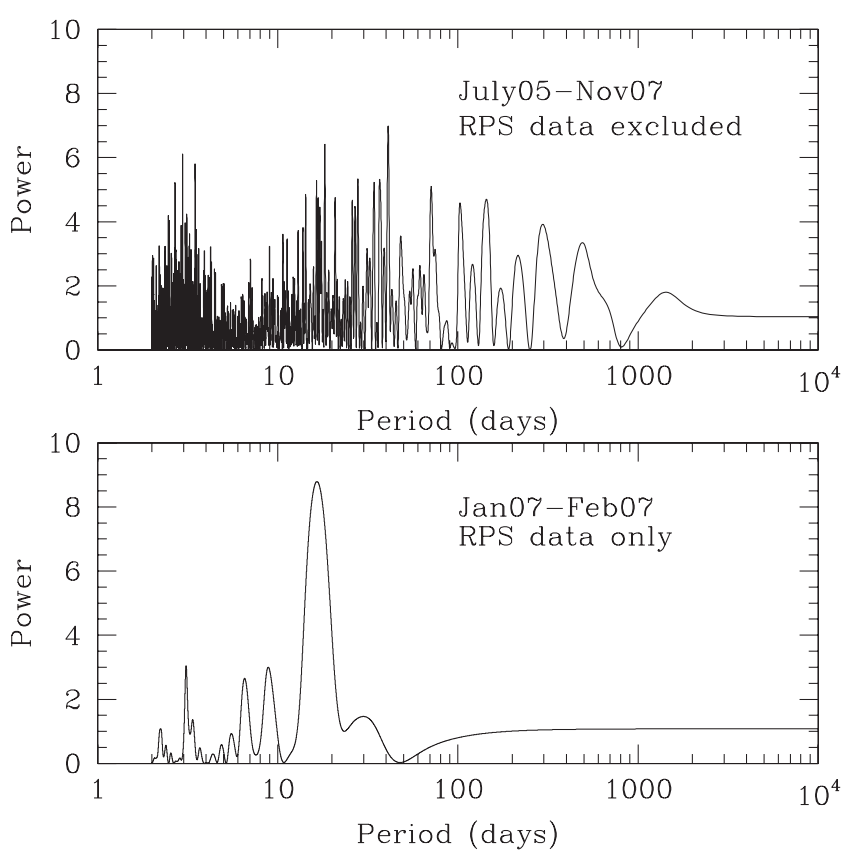

Figure 2. Periodogram of radial-velocity data for HD 16417, which hosts a Neptune-mass planet with a period of 17 days. Top: Rocky Planet Search (RPS) data excluded $(N=26)$; there are no significant periodicities. Bottom: Rocky Planet Search data only $(N=24)$. The planetary signal is clearly evident. High-cadence observations during the Rocky Planet Search were critical in detecting this low-mass planet.

Earth-like planets, only the radial-velocity method targets the nearest stars. Planets detected by this method will therefore be most amenable to follow-up characterisation, with the potential to vastly enhance our knowledge of planet formation.

A major innovation of this project is that we are able to observe the Alpha Centauri system throughout the night, year-round. There is no other facility equipped with a highresolution spectrograph from which this can be done. Mt. John is at a latitude of $44^{\circ}$ south, making Alpha Centauri circumpolar: it is always observable (minimum altitude 15 degrees above the horizon). We are thus in a unique position to optimally sample periods close to one year. It is critical to control the observing window in this manner in order to detect or exclude low-amplitude signals (Tinney et al. 2011). This was demonstrated by our detection of the 17 Earth-mass planet HD 16417b (O'Toole et al. 2009a). The discovery was made possible by the "Rocky Planet Search" strategy which employed continuous 48-night observing blocks. The power of this intensive observing approach is most dramatically demonstrated by our detection of HD $16417 \mathrm{~b}$ from 24 epochs obtained in that 48-night run, and the non-detection of the same planet (Figure 2) in a similar quantity of similar quality data spread over a 2-year period (O'Toole et al. 2009a,b). To detect extremely low-mass planets, observations must be made as continuously as possible. Mt. John's southern location means that while other observatories are limited to observe Alpha Centauri for 9 or 10 months, we can obtain radial-velocity data for 12 months a year. 


\section{Summary}

We are using the HERCULES spectrograph at the McLellan 1-m telescope at Mt. John Observatory in New Zealand to perform the world's most intensive radial-velocity observing campaign, targeting the nearest stars Alpha Centauri A \& B. This site has three critically important advantages: (1) we can observe Alpha Centauri year-round, (2) the proven ability to obtain high-precision radial velocities, and (3) the availability of a large amount of observing time. This project directly addresses a significant strategic goal from both Australia's Decadal Plan ("how common are planetary systems and conditions suitable for life?") and NASA's Science Plan ("to establish a comprehensive census of the planetary systems in our solar neighborhood and to determine whether Earth-mass planets are common or rare"). At the completion of this program, even if no planets are found, we will have stringent limits for planets around these two very nearby stars. This will be highly valuable a priori information for the planning and design of any next generation space telescope, with the goal to detect, characterise, and even search for bio-signatures in Earth-like worlds around nearby stars. Any future Terrestrial Planet Finder-type mission will likely have Alpha Centauri on its target list. Advance information on the presence or absence of terrestrial planets in this system can play an important role in the overall design of such a mission. If we do find signs of Earth-mass planets orbiting our closest neighbour, then a smaller and much less costly version of Terrestrial Planet Finder could be built. Finally, we draw attention to the promising results of Dumusque et al. (2012), who report the detection of an Earth-mass planet in a 3.236-day orbit around Alpha Centauri B (though this is controversial, e.g. Hatzes 2013). That result, if confirmed by independent data from this and other programs, would suggest that further low-mass planets may orbit in the Alpha Centauri system, in light of the findings from Kepler that systems of multiple terrestrial-mass planets seem to be common (Howard et al. 2012, Batalha et al. 2012).

\section{References}

Ayres, T. R. 2009, ApJ, 696, 1931

Batalha, N. M., Rowe, J. F., Bryson, S. T., et al. 2012, ApJS, 204, article id. 24

Bedding, T. R., Kjeldsen, H., Butler, R. P., et al. 2004, ApJ, 614, 380

Borucki, W. J., Koch, D., Basri, G., et al. 2010, Science, 327, 977

Butler, R. P., Bedding, T. R., Kjeldsen, H., et al. 2004, ApJL, 600, L75

Cochran, W. D., \& Hatzes, A. P. 1996, Ap \&S SS, 241, 43

Dumusque, X., Pepe, F., Lovis, C., et al. 2012, Nature, 491, 207

Duquennoy, A. \& Mayor, M. 1991, A\&A, 248, 485

Endl, M., Kürster, M., \& Els, S. 2000, A\&A, 362, 585

Endl, M., Robertson, P., Cochran, W. D., et al. 2012, ApJ, 759, 19

Gautier, T. N., III, Batalha, N. M., Borucki, W. J., et al. 2010, arXiv:1001.0352

Gould, A., Udalski, A., An, D., et al. 2006, ApJL, 644, L37

Guedes, J. M., Rivera, E. J., Davis, E., et al. 2008, ApJ, 679, 1582

Haghighipour, N. \& Raymond, S. N. 2007, ApJ, 666, 436

Hatzes, A. P. 2013, ApJ, 770, 133

Hearnshaw, J. B., Barnes, S. I., Kershaw, G. M., Frost, N., Graham, G., \& Ritchie, R., Nankivell G. R. 2002, Exp. Astron., 13, 59

Hearnshaw, J. B., Barnes, S. I., \& Endl, M. 2009, Proc. 10th Asian-Pacific Regional IAU Meeting 2008,350 .

Heintz, W. D. 1982, The Observatory, 102, 42

Howard, A. W., Marcy, G. W., Bryson, S. T., et al. 2012, ApJS, 201, 15

Ida, S. \& Lin, D. N. C. 2004, ApJ, 604, 388 
Kjeldsen, H., Bedding, T. R., Butler, R. P., et al. 2005, ApJ, 635, 1281

Kürster, M., Endl, M., Els, S., et al. 2000, A\& $\&$, 353, L33

Mayor, M., Udry, S., Lovis, C., et al. 2009, A\&A, 493, 639

Murdoch, K. A., Hearnshaw, J. B., \& Clark, M. 1993, ApJ, 413, 349

Narayan, R., Cumming, A., \& Lin, D. N. C. 2005, ApJ, 620, 1002

O'Toole, S., Tinney, C. G., Butler, R. P., et al. 2009a, ApJ, 697, 1263

O'Toole, S. J., Jones, H. R. A., Tinney, C. G., et al. 2009b, ApJ, 701, 1732

Pourbaix, D., Neuforge-Verheecke, C., \& Noels, A. 1999, A\&A A, 344, 172

Pourbaix, D., Nidever, D., McCarthy, C., Butler, R. P., Tinney, C. G., Marcy, G. W., Jones, H. R. A., Penny, A. J., Carter, B. D., Bouchy, F., Pepe, F., Hearnshaw, J. B., Skuljan, J., Ramm, D., \& Kent, D. 2002, A\&A, 386, 280

Queloz, D., Henry, G. W., Sivan, J. P., et al. 2001, A\&A, 379, 279

Quintana, E. V., Lissauer, J. J., Chambers, J. E., \& Duncan, M. J. 2002, ApJ, 576, 982

Quintana, E. V. \& Lissauer, J. J. 2006, Icarus, 185, 1

Quintana, E. V., Adams, F. C., Lissauer, J. J., \& Chambers, J. E. 2007, ApJ, 660, 807

Robertson, P., Endl, M., Cochran, W. D., et al. 2012, ApJ, 749, 39

Thébault, P., Marzari, F., \& Scholl, H. 2008, MNRAS, 388, 1528

Thébault, P., Marzari, F., \& Scholl, H. 2009, MNRAS, 393, L21

Tinney, C. G., Wittenmyer, R. A., Butler, R. P., et al. 2011, ApJ, 732, 31

Walker, G. A. H., Walker, A. R., Irwin, A. W., et al. 1995, Icarus, 116, 359

Wittenmyer, R. A., Endl, M., Wang, L., et al. 2011, ApJ, 743, 184

Xie, J.-W., Zhou, J.-L., \& Ge, J. 2010, ApJ, 708, 1566

Xie, J.-W., Zhou, J.-L., \& Ge, J. 2011, European Physical Journal Web of Conferences, 16, 5003

Zhou, J.-L., Aarseth, S. J., Lin, D. N. C., \& Nagasawa, M. 2005, ApJL, 631, L85 